\title{
EDITORIAL
}

\section{When research goes wrong: the importance of clinical trials methodology}

\author{
Pediatric Research (2020) 88:518-519; https://doi.org/10.1038/s41390- \\ 020-0984-5
}

\section{NEW CLINICAL TRIALS METHODOLOGY}

Clinical trials methodology is constantly being developed and refined. Consensus statements from the CONSORT (CONsolidated Standards Of Reporting Trials) guidelines on clinical trial reporting and the registering of study protocols online (e.g., clinical trials.gov) has become a standard practice. This transparency and ethical reviews of clinical trial protocols should protect participants in these trials but needs constant revision and refinement. Therefore, careful adherence is needed to evolving clinical research methodology as exemplified in the Enhancing the QUAlity and Transparency Of health Research (EQUATOR) network. EQUATOR is an international initiative that aims to improve the value and reliability of health research literature by promoting accurate and transparent reporting (https://www.equator-network.org/). It provides links to the reporting guidelines for the main study types, for example, CONSORT (for randomized controlled trials; https://www.equator-network.org/ reporting-guidelines/consort/) and PRISMA (Preferred Reporting Items for Systematic Reviews and Meta-Analyses; https://www. equator-network.org/reporting-guidelines/prisma/). These reporting guidelines are also highlighted in the instructions for authors of Pediatric Research because they are vital for future research synthesis.

In this issue of Pediatric Research, the importance of clearly defining control participants in studies to allow accurate comparisons is highlighted. Zaslawski et al. ${ }^{1}$ in this issue of the journal also highlight the need for rigorous reporting of controls. They demonstrate the utility of the existing reporting tool (TIDieR; Template for Intervention Description and Replication), which they had modified. This tool includes 12 items: name that describes the arm, procedures, references for justification, materials, specific training provided, a description of who provided the intervention, locations of the intervention, route of delivery, number of delivered interventions, and description of personalization or modification if any occurred. This use of this modified TIDieR tool aims to improve the reproducibility and implementation of clinical trials in children.

In addition, the development of the SPIRIT (Standard Protocol Items for Randomlzed Trials) (https://www.spirit-statement.org/ about-spirit/) guidelines is to help improve the completeness and quality of trial protocols. The evidence-based SPIRIT recommendations were developed using systematic, transparent methodology and broad consultation with 115 experts representing diverse stakeholders involved in the design, funding, conduct, review, and publication of trial protocols. These recommendations have recently been expanded to include children as the SPIRIT-C guidelines. The evidence synthesis has been completed, the Delphi survey and international consensus have been finished, and the checklists have been finalized for SPIRIT-C ${ }^{2}$ (https://lab.research.sickkids.ca/enrich/ research-projects/spirit-c/). This information will improve standardization of the way clinical trials in children are conducted using SPIRIT-C and aims to improve the impact and benefit of clinical research for children. ${ }^{2}$

WHY IS THIS SO IMPORTANT: LESSONS FROM THE PAST

As an example of what can happen if correct methodology is not used is the continued perception that vaccines, specifically the measles, mumps, and rubella (MMR), causes autism. The proposed mechanism was that intestinal inflammation occurred following the vaccine which allowed intestinal peptides to enter the blood stream and impair brain development. The original observation, published in The Lancet, ${ }^{3}$ reported on 8 children who developed autism within a month of vaccination. This study was critically flawed by the methodology used: there was no control arm (children vaccinated who did not develop autism), and the intestinal symptoms occurred after the symptoms of autism, not before (https://www.chop.edu/ centers-programs/vaccine-education-center/vaccines-and-otherconditions/vaccines-autism).

In a second publication attempting to link autism with the measles virus, the authors studied 91 patients with autism and found 75 of them had measles virus fusion and hemagglutinin genes in their intestinal tissue by reverse transcription-polymerase chain reaction (RT-PCR) and Nucleocapsid gene by RT in situ PCR, which the authors interpreted as suggesting an association between the presence of measles virus and autism. ${ }^{4}$ This study too had critical flaws in methodology. Measles vaccine virus is live and attenuated. It is plausible that, following the MMR, a child may have this measles form in intestinal tissue. The control arm for this study, children without autism, was not matched to the children with autism in terms of immunization status (did or did not get the vaccine) and the length of time between being vaccinated and having intestinal samples taken. Therefore, the specificity of this finding for autism was not shown. The authors also failed to distinguish between natural measles virus and the attenuated form used in vaccines, so the conclusion that the virus in the intestinal tissue came from the vaccine is unsupported. The RT-PCR method to detect virus is very sensitive and would be expected to produce false positives, but how this was avoided in the study was not described. And no mention is made if the person doing the assays was blinded (https://www.chop. edu/centers-programs/vaccine-education-center/vaccines-andother-conditions/vaccines-autism). Thus we have two studies failing to meet methodologic standards that have perpetuated the lay public's fear of vaccines, to the detriment of children.

\section{FUTURE DIRECTIONS}

Should all medical students learn about these studies to ensure these mistakes are never repeated? This example makes us look at our research practice, which continues to change and may be judged negatively in the future using a different lens. In this study, inadequate study design to include appropriate controls has led to long held public beliefs. The loss of trust in medical research 
communities as a result of this study may have negatively affected vulnerable populations as they may not receive vaccinations. ${ }^{5}$

As pediatricians and child health researchers, we represent some of the most vulnerable and socioeconomically deprived members of society who, in addition, have no vote, a fact that adds additional responsibility to us to develop the highest standards of collaborative research. ${ }^{6}$ These tools are vital to protect families and researchers as they strive together to improve health outcomes.

\section{ACKNOWLEDGEMENTS}

This study was supported by the National Children's Research Centre, Crumlin, Dublin, Ireland and Health Research Board Ireland (to E.J.M.) and NIH/NICHD P01HD085928 (to C.F.B.).

\section{AUTHOR CONTRIBUTIONS}

E.J.M. and C.F.B.: substantial contributions to conception and design, revising the article critically for important intellectual content, and final approval of the version to be published.

\section{ADDITIONAL INFORMATION}

Competing interests: The authors declare no competing interests.

Publisher's note Springer Nature remains neutral with regard to jurisdictional claims in published maps and institutional affiliations.
Eleanor J. Molloy ${ }^{1,2,3,4,5}$ and Cynthia F. Bearer ${ }^{6,7}$

${ }^{1}$ Discipline of Paediatrics, Trinity College, the University of Dublin, Dublin, Ireland; ${ }^{2}$ Children's Health Hospital (CHI) at Tallaght, Tallaght University Hospital, Dublin, Ireland; ${ }^{3}$ Trinity Translational Medicine Institute, St James Hospital, Dublin, Ireland; ${ }^{4}$ Neonatology, $\mathrm{CHI}$ at Crumlin, Dublin, Ireland; ${ }^{5}$ Paediatrics, Coombe Women's and Infant's University Hospital, Dublin, Ireland; ${ }^{6}$ Division of Neonatology, Department of Pediatrics, Rainbow Babies \& Children's Hospital, Cleveland, $\mathrm{OH}, \mathrm{USA}$ and ${ }^{7}$ Case Western Reserve University School of Medicine, Cleveland, $\mathrm{OH}$, USA

Correspondence: Eleanor J. Molloy (eleanor.molloy@tcd.ie)

\section{REFERENCES}

1. Zaslawski, Z., Balasubramanaiam, B. \& Kelly, L.E. Is your standard, my standard? Why control arm reporting matters in pediatric clinical trials. Pediatr Res (2020). https://doi.org/10.1038/s41390-020-0974-7.

2. Clyburne-Sherin, A. V. et al. Recommendations and evidence for reporting items in pediatric clinical trial protocols and reports: two systematic reviews. Trials 16, 417 (2015).

3. Wakefield, A. J. et al. Ileal-lymphoid-nodular hyperplasia, non-specific colitis, and pervasive developmental disorder in children. Lancet 351, 637-641 (1998).

4. Uhlmann, V. et al. Potential viral pathogenic mechanism for new variant inflammatory bowel disease. Mol. Pathol. 55, 84-90 (2002).

5. Gupta, K., Chen, M. \& Joshua Rocker, J. Measles: taking steps forward to prevent going backwards. Curr. Opin. Pediatr. 32, 436-445 (2020).

6. Molloy, E. J., Mader, S., Modi, N. \& Gale, C. Parent, child and public involvement in child health research: core value not just an optional extra. Pediatr. Res. 85, 2-3 (2019). 